\section{DGAI-Sektionen und -Arbeitsgemeinschaften}

$\begin{array}{ll}\text { Sektionen } & \text { Sprecher } \\ \text { Dermatologie } & \text { Przybilla } \\ \text { HNO } & \text { Bachert } \\ \text { Immunologie } & \text { Saloga } \\ \text { Pädiatrie } & \text { Bauer } \\ \text { Pneumologie/Innere Medizin } & \text { Bergmann } \\ \text { Umwelt/Arbeitsmedizin } & \text { Behrendt } \\ \text { Arbeitsgemeinschaften } & \text { Sprecher } \\ \text { Exogen allergische Alveolitis } & \begin{array}{l}\text { Müller-Wening } \\ \text { (gemeinsam mit DGP) }\end{array} \\ \text { Insektengiftallergie } & \text { Przybilla } \\ \text { Komplementärmethoden } & \text { (gemeinsam mit ADF) } \\ \text { Nahrungsmittelallergie } & \text { Dorsch } \\ \end{array}$

immer noch als beschämend niedrig bezeichnet werden muss.

\section{Aktivitäten der DGAI}

Während sich in früheren Jahren die Arbeit der DGAI vorwiegend auf die Organisation von Tagungen sowie auf die Bemühungen um eine Zeitschrift beschränkte, sind heute auch standespolitische Aufgaben, Pressearbeit sowie die Erarbeitung von Positionspapieren, Leitlinien und Stellungnahmen wesentliche Schwerpunkte der Gesellschaftsaktivität. Diese wird nicht mehr nur vom
Vorstand allein getragen, sondern wesentlich von den Sektionen und Arbeitsgemeinschaften.

\section{Literatur \\ 1. Fuchs E. Zur Erinnerung an Karl Hansen. MMW 1963; 105: 1961 \\ 2. Kikuth W. Zum Geleit. Allergie 1 - Organ der Deutschen Gesellschaft für Allergiefor- schung (Beilage): In: Dtsch Med Wschr 1952; $77: 828$}

3. Schadewaldt H, Fuchs E: Geschichte der Deutschen Gesellschaft für Allergie- und Immunitätsforschung 1951-1984. München: Dustri-Verlag, 1994

4. Schadewaldt H. Geschichte der Allergie. Bd. 1-4. München, Dustri-Verlag 1979-1982

Prof. Dr. G. Schultze-Werninghaus, Prof. Dr. Dr. J. Ring folgte eine Beitragserhöhung auf 140,00 Mark, die jedoch den Bezug der Fachzeitschrift ALLERGO JOURNAL mit einschließt. Im kommenden Jahr wird die Umstellung auf Euro eine neue Anpassung erforderlich machen.

Während bis vor kurzem die administrative Arbeit der Gesellschaft von den Vorstandsmitgliedern alleine erledigt worden war, gelang es seit 1999 eine Geschäftsstelle der DGAI einzurichten, die von Frau Erika Ratzinger hervorragend betreut wird. Dies wurde nur möglich aufgrund längerfristiger Vereinbarungen mit kompetenten und allergologisch aktiven Industriepartnern. Diese Hauptsponsoren der DGAI sind derzeit:

- Aventis Behring GmbH (ursprüng-

lich Hoechst Pharma und Rhone Poulenc Rorer)

- Alk-Scherax Arzneimittel GmbH

- Allergopharma Joachim Ganzer KG

- Bencard Allergie GmbH

— Essex Pharma GmbH

- HAL Allergie GmbH

- Novartis Pharma GmbH/Novartis

Consumer Health GmbH

- Pharmacia GmbH \& Co KG

- UCB Pharma GmbH

Die Mitgliederzahl der Gesellschaft hat heute mit über 700 Beitragszahlern einen Höchststand erreicht, der allerdings in Anbetracht der mehr als 5.000 allergologisch tätigen Ärzte in Deutschland

\title{
Nachruf auf Prof. Hermann Michel
}

\author{
Im Juli 2001 ist Professor Dr. med. Hermann Michel in Berlin im 84. \\ Lebensjahr verstorben. Michel ist wahrscheinlich nur den Älteren \\ unter den Lesern vertraut. Schaut man aber in die Kongressunterla- \\ gen früherer Jahre, so wird rasch klar, warum Michel zu den Grün- \\ dervätern der deutschen Allergologie und Immunologie gehört.
}

1 ermann Michel wurde in der allergologischen Literatur vor allem verankert durch seine Mitherausgeberschaft bei der dritten Auflage des Werkes von Hugo Kämmerer und Hermann Michel: Allergische Diathese und allergische Erkrankungen, 3. Auflage, erschienen bei Bergmann, München 1956.

Michel hat aber nicht nur mit dem Internisten Hugo Kämmerer, München (1878-1969), einem der Gründer der Deutschen Gesellschaft für Allergologie und klinische Immunologie 1951, zusammengearbeitet. Er hat vielfach die Aktivitäten der Deutschen Gesellschaft für Allergologie und klinische Immunologie unterstützt, z. B. allergologische Tagungen in Berlin während der 50er und 60er Jahre organisiert. Auf der 14. Tagung der DGAI 1979 in Freiburg hielt Michel die Festrede zum 100. Geburtstag von Kämmerer.

Professor Michel war Internist. Er war als klinischer Allergologe in Berlin tätig, am Klinikum Steglitz, in der Medizinischen Klinik und Poliklinik. Sein wissenschaftliches Interesse galt unter anderem der Insulin-Allergie. Er hat hierzu eine Reihe von internationalen Publikationen beigetragen, so in Acta allergologica (heute: Allergy) 1961; 16: 1. Darüber hinaus hat Michel sich mit anderen Medikamenten-Allergien, Kollagenosen, der spezifischen Immuntherapie und weiteren internistisch-allergologischen Fragestellungen beschäftigt.

Hermann Michel war über viele Jahre interessierter Teilnehmer nationaler und internationaler Kongresse auch nach dem Ende seiner beruflichen Laufbahn und ist auf diese Weise vielen in lebhafter Erinnerung, ebenso wie seine Ehefrau Marianne Michel-Rudolph, der unser Mitgefühl gilt. Die Deutsche Gesellschaft für Allergologie und klinische Immunologie wird ihm ein ehrendes Gedenken bewahren.

Prof. Dr. G. Schultze-Werninghaus 